\title{
A New Method for Designing Modified Compact Microstrip LPF with Sharp Roll-Off and Wide Stopband
}

\author{
Abbas HOSEINABADI ${ }^{1}$, Mohammad Bagher TAVAKOLI ${ }^{2}$, Mohammad Jalal RASTEGAR FATEMI ${ }^{1}$, \\ Farbod SETOUDEH ${ }^{3}$
}

\footnotetext{
${ }^{1}$ Dept. of Electronics, Coll. of Engineering, Saveh Branch, Islamic Azad University, Saveh, Iran

${ }^{2}$ Dept. of Electronics, Coll. of Engineering, Arak Branch, Islamic Azad University, Arak, Iran

${ }^{3}$ Dept. of Electrical Engineering, Arak University of Technology, Arak, Iran
}

\{a-hosseinabadi,m-tavakoli\}@iau-arak.ac.ir,j_rastegar@iau-saveh.ac.ir, f.setoudeh@arakut.ac.ir

Submitted June 3, 2021 / Accepted October 24, 2021

\begin{abstract}
A new method for designing a compact microstrip lowpass filter (LPF) with wide stopband width $(S B W)$ and sharp roll off (ROF) is presented. In the proposed designing procedure, high impedance microstrip lines are bent to achieve an LPF with compact size. Then, to compensate for the effect of bending microstrip lines, the lengths of the lines are mathematically modified. Moreover, adding a suppressing cell composed of a radial stub resonator and a butterfly stub resonator increases the $S B W$. Also, an elliptic filter structure is used to obtain sharp ROF. In this work, an LPF with $1.12 \mathrm{GHz}$ cutoff frequency, $0.147 \lambda_{\mathrm{g}} \times 0.133 \lambda_{\mathrm{g}}$ filter size; where $\lambda_{\mathrm{g}}$ is the guided wavelength at cutoff frequency, the $S B W$ equal to $13.4 \mathrm{GHz}$, and the ROF more than $201 \mathrm{~dB} / \mathrm{GHz}$, is designed, simulated and fabricated to demonstrate efficiency of the proposed method. Also, the other conventional characteristics for the fabricated LPF such as $0.3 \mathrm{~dB}$ insertion loss, $14.4 \mathrm{~dB}$ return loss, and suppression factor equal to 2.2 , are in the appropriate range of their amounts.
\end{abstract}

\section{Keywords}

Lowpass filter, microstrip, elliptic filter, stopband width, roll off, compact size

\section{Introduction}

In recent decades, the communication systems, especially the microwave applications, benefited from the microstrip filters. The lowpass filters (LPFs) are widely used in most of the communication systems to reject unwanted high frequency signals from system answers [1], [2]. Newly, researchers tried to improve microstrip LPF characteristics such as increasing relative stopband bandwidth (RSB), sharpening roll off (ROF), compacting LPF normalized circuit size (NCS), obtaining low insertion loss (IL) and high return loss (RL) in the passband region, and enhancing suppression factor (SF) in the stopband zone; whereas the ROF, the NCS and the RSB were more con- sidered [3-21]. It should be noted that the RSB, NCS, ROF and SF parameters are commonly used for the LPF and are defined in article [3]. To obtain the high ROF in an LPF that is proposed in [4], stepped impedance modified T-type with stepped impedance stub is used, but it does not have high RSB and the IL is not in the appropriate range. In [5] an LPF using T-shaped resonators is presented to achieve a sharp transition skirt; however its RSB is very small. In [6], the proposed LPF is comprised of three coupled stepped impedance resonators to create the high ROF, while one of disadvantages of this work is the low SF. In some of the reviewed articles, the NCS is more considered. In [7], a folded three-element LPF is proposed to achieve compact size, but its ROF is very low. In other two works [8], [9] the feeding lines are folded to reduce filter size, nevertheless in [9] the RSB is not high and in [8] the ROF is middle and the RSB is also low. Considering the importance of the RSB in the LPF many of the recent papers try to improve the RSB [10-18]. In [10-12] by using stepped impedance resonators the LPFs are designed, and although those have good RSB, their ROFs are very low. In another article [13], the LPF structure is composed of a defected rectangular-shaped resonator and a rectangular shape resonator as a suppressing cell, and amount of its RSB is high, also its NCS is low, nevertheless its ROF is not appropriate. An LPF with wide stopband and low NCS using triangular-shaped resonator is presented in [14], but its ROF is low. Other structures which are used for LPF designing include defected ground structure (DGS) to improve the RSB [15-18]. It is true that using the DGS improves the stopband width, but it complicates filter designing. Also the LPFs that are presented in [15-18] have not high ROF. In another effort an LPF with ultra-wide stopband is proposed [19], while its ROF is moderate and its IL is not acceptable and it also has a complex design. In article [20], using a meandered semi-hairpin resonator which is combined with a suppressing unit, an LPF with wide stopband and small NCS is presented and the disadvantages of it are small ROF and complexity in filter design. In [3], an LPF with good ROF and RSB consisting of two resonators with different triangular patches and four 
high-low impedance resonators as suppressing cells has been designed. Finally, in [21], another LPF with good ROF and RSB consisting of three cascaded resonators with different semi-circle patches and four suppressors employing radial stubs has been proposed. But in the last two filters, the NCS is relatively large and those have complex design, too.

In this paper, the use of an elliptic filter structure causes a high ROF. To achieve miniature filter size, the high impedance microstrip lines are bent and their lengths are modified by mathematical analysis. Then as a result of adding suppressing cell, which it consists of a combination of a radial stub resonator and a butterfly stub resonator, the RSB increases. At last, to show efficiency of the designed LPF, it was fabricated and measured.

\section{LPF Design Process}

\subsection{Conventional Elliptic LPF Design}

Due to the desired characteristics of an elliptic filter such as high ROF, high SF, and low IL, a six-pole elliptic LPF with $0.1 \mathrm{~dB}$ passband ripples and $1.1 \mathrm{GHz}$ cutoff frequency is introduced. Figure la shows the LPF prototype circuit with lumped elements. In Fig. 1b layout of the six-pole microstrip LPF is depicted. All inductors and capacitors are realized using high-impedance and lowimpedance lines respectively. Characteristic impedance for high-impedance lines $Z_{0 \mathrm{~L}}=131 \Omega$, and characteristic impedance for low-impedance lines $Z_{0 \mathrm{C}}=20 \Omega$ are selected. In addition, to calculate high-impedance line lengths $l_{\mathrm{L} i}$ for $i=1,2, \ldots, 5$ and low-impedance line lengths $l_{\mathrm{C} j}$ for $j=2,4,6$, substituting the corresponding parameters from Tab. 1 in (1) is used [2]:

$$
\begin{aligned}
& l_{\mathrm{L} i}=\frac{\lambda_{\mathrm{gL}}}{2 \pi} \sin ^{-1}\left(\frac{2 \pi f_{\mathrm{c}} L_{i}}{Z_{0 \mathrm{~L}}}\right), \\
& l_{\mathrm{C} j}=\frac{\lambda_{\mathrm{gC}}}{2 \pi} \sin ^{-1}\left(2 \pi f_{\mathrm{c}} Z_{0 \mathrm{C}} C_{j}\right)
\end{aligned}
$$
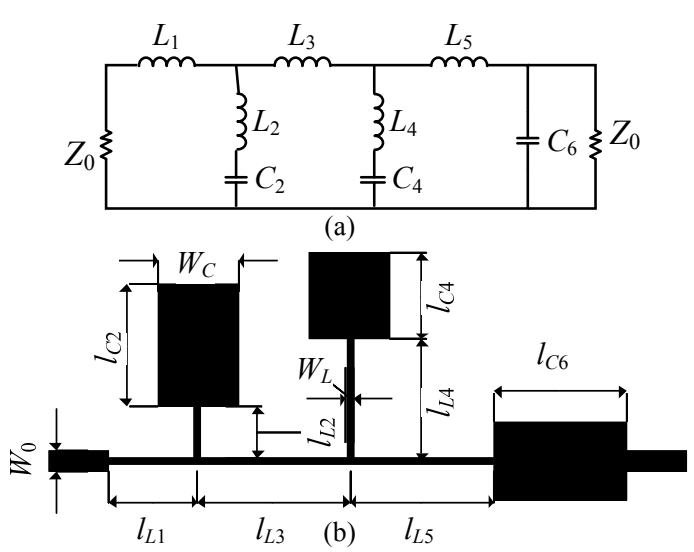

Fig. 1. The conventional elliptic LPF: (a) The prototype circuit with lumped elements. (b) Layout.

\begin{tabular}{|c|c|c|c|c|c|c|c|}
\hline Sym. & Value & Sym. & Value & Sym. & Value & Sym. & Value \\
\hline$L_{1}$ & 4.7378 & $L_{2}$ & 3.3256 & $L_{3}$ & 7.9020 & $L_{4}$ & 6.7163 \\
\hline$L_{5}$ & 7.5280 & $C_{2}$ & 2.9041 & $C_{4}$ & 2.2371 & $C_{6}$ & 2.9557 \\
\hline$l_{\mathrm{L} 1}$ & 7.2 & $l_{\mathrm{L} 2}$ & 5 & $l_{\mathrm{L} 3}$ & 12.2 & $l_{\mathrm{L} 4}$ & 10.3 \\
\hline$l_{\mathrm{L} 5}$ & 11.6 & $l_{\mathrm{C} 2}$ & 10.5 & $l_{\mathrm{C} 4}$ & 8 & $l_{\mathrm{C} 6}$ & 10.7 \\
\hline$W_{0}$ & 1.86 & $W_{\mathrm{L}}$ & 0.2 & $W_{\mathrm{C}}$ & 6.5 & $Z_{0}$ & 50 \\
\hline$\lambda_{\mathrm{gL}}$ & 178.4 & $\lambda_{\mathrm{gC}}$ & 158.4 & $f_{\mathrm{p} 1}$ & 1.298 & $f_{\mathrm{p} 2}$ & 1.619 \\
\hline
\end{tabular}

Tab. 1. The values of inductors $[\mathrm{nH}]$, capacitors $[\mathrm{pF}], \mathrm{I} / \mathrm{O}$ characteristic impedance $[\Omega]$, poles frequency [GHz], wavelengths $[\mathrm{mm}]$, widths $[\mathrm{mm}]$ and lengths $[\mathrm{mm}]$ related to Fig. 1 .

where $f_{\mathrm{c}}=1.1 \mathrm{GHz}$ is the desired cutoff frequency and $\lambda_{\mathrm{gL}}$ and $\lambda_{\mathrm{gC}}$ are guided wavelengths for high-impedance and low-impedance lines at $f_{\mathrm{c}}$ respectively.

For the LPF with lumped elements two finite-frequency attenuation poles occur at $f_{\mathrm{p} 1}$ and $f_{\mathrm{p} 2}$ that are presented by (2). The values of $f_{\mathrm{p} 1}$ and $f_{\mathrm{p} 2}$ are also given in Tab. 1 along with other related values in Fig. 1.

$$
\begin{aligned}
& f_{\mathrm{p} 1}=\frac{1}{2 \pi \sqrt{L_{4} C_{4}}}, \\
& f_{\mathrm{p} 2}=\frac{1}{2 \pi \sqrt{L_{2} C_{2}}} .
\end{aligned}
$$

The compensating for the effect of connecting inductor $L_{2}$ to capacitor $C_{2}$ which is a discontinuity with different widths, the simultaneous compensation for the unwanted susceptance at the junction of the inductive lines for $L_{1}, L_{2}$, and $L_{3}$, is achieved by correcting $l_{\mathrm{L} 2}$ and $l_{\mathrm{C} 2}$, while $l_{\mathrm{L} 1}$ and $l_{\mathrm{L} 3}$ are kept unchanged. For this purpose, equation (3) is solved by substituting the cutoff frequency $f_{\mathrm{c}}$ and the attenuation pole frequency $f_{\mathrm{p} 2}$ instead of $f[2]$ :

$$
\frac{1}{2 \pi f L_{2}-\left(2 \pi f C_{2}\right)^{-1}}=B_{\mathrm{b} 2}(f)+B_{\mathrm{T}}(f)
$$

where according to Fig. $1 \mathrm{~b}, B_{\mathrm{b} 2}(f)$ specifies the susceptance of $L_{2}$ and $C_{2}$ branch which was implemented by microstrip lines and will be obtained by (4):

$$
\begin{gathered}
B_{\mathrm{b} 2}(f)=\frac{1}{X_{\mathrm{L} 2}(f)-\frac{1}{B_{\mathrm{C} 2}(f)}}, \\
X_{\mathrm{L} 2}(f)=Z_{0 \mathrm{~L}} \sin \left(\frac{2 \pi l_{\mathrm{L} 2}}{\lambda_{\mathrm{gL}}(f)}\right)+Z_{0 \mathrm{C}} \tan \left(\frac{\pi l_{\mathrm{C} 2}}{\lambda_{\mathrm{gC}}(f)}\right), \\
B_{\mathrm{C} 2}(f)=\frac{1}{Z_{0 \mathrm{C}}} \sin \left(\frac{2 \pi l_{\mathrm{C} 2}}{\lambda_{\mathrm{gC}}(f)}\right)+\frac{1}{Z_{0 \mathrm{~L}}} \tan \left(\frac{\pi l_{\mathrm{L} 2}}{\lambda_{\mathrm{gL}}(f)}\right) .
\end{gathered}
$$

Note that the second term on the right in (5) shows the effect of low impedance line in the step connection related to $L_{2}$ and $C_{2}$ branch. Similarly, the second term on the right in (6) shows the effect of high impedance line in the step connection related to $L_{2}$ and $C_{2}$ branch. In (3) $B_{\mathrm{T}}(f)$ is unwanted susceptance resulting $\mathrm{T}$-junction between three high impedance lines $l_{\mathrm{L} 1}, l_{\mathrm{L} 2}$ and $l_{\mathrm{L} 3}$ and it can be written as (7): 


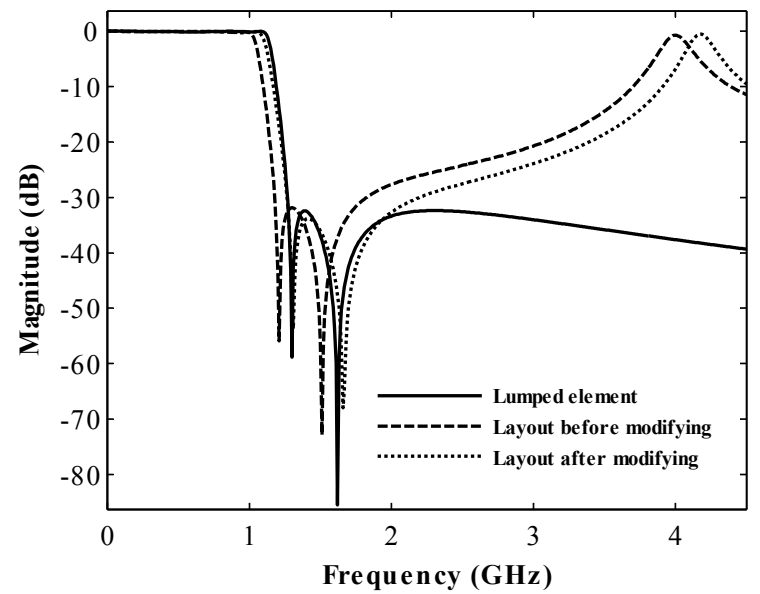

Fig. 2. The simulated $S_{21}$ parameters for the LPF with lumped elements and for the LPF with microstrip layout before and after correcting by (3).

$$
\begin{array}{r}
B_{\mathrm{T}}(f)=\frac{1}{Z_{0 \mathrm{~L}}} \tan \left(\frac{\pi l_{\mathrm{L} 1}}{\lambda_{\mathrm{gL}}(f)}\right)+\frac{1}{Z_{0 \mathrm{~L}}} \tan \left(\frac{\pi l_{\mathrm{L} 2}}{\lambda_{\mathrm{gL}}(f)}\right)+ \\
\frac{1}{Z_{0 \mathrm{~L}}} \tan \left(\frac{\pi l_{\mathrm{L} 3}}{\lambda_{\mathrm{gL}}(f)}\right) .
\end{array}
$$

Also for $L_{4}$ and $C_{4}$ branch an equation similar to (3) should be written by replacing the parameters corresponding to it then solved at $f_{\mathrm{c}}$ and $f_{\mathrm{p} 1}$. After correcting, the lengths of the corrected lines are obtained as $l_{\mathrm{L} 2}=4 \mathrm{~mm}$, $l_{\mathrm{C} 2}=10.7 \mathrm{~mm}, l_{\mathrm{L} 4}=9.2 \mathrm{~mm}$ and $l_{\mathrm{C} 4}=7.7 \mathrm{~mm}$. In Fig. $2 \mathrm{~S}_{21}$ parameter responses for the LPF with lumped elements and microstrip layout before and after correcting by (3) are illustrated. As shown in Fig. 2 locations of $f_{\mathrm{p} 1}$ and $f_{\mathrm{p} 2}$ in the LPF with microstrip layout after correcting by (3) and the LPF with lumped elements are almost matched.

\subsection{Compacting LPF Size}

The dimensions of the filter can be reduced by using the technique of bending high impedance microstrip lines. Of course, it should be noted that bending the microstrip lines causes unwanted effects on the values of inductors and capacitors implemented with microstrip lines. To correct the deviation in the inductor and the capacitor values, which means deviation in electrical length of microstrip lines, the physical length of the lines must be modified. For example, Figure 3 shows how $l_{\mathrm{L} 2}$ and $l_{\mathrm{L} 3}$ are bent to reduce the filter size.

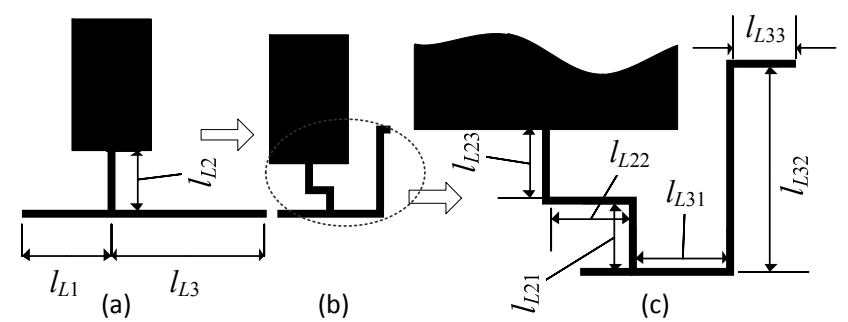

Fig. 3. Bending microstrip lines to reduce filter size: (a) Before bending, (b) after bending, (c) detailed.
To modify the deviation caused by bending of $l_{\mathrm{L} 2}$ and $l_{\mathrm{L} 3}$, susceptance $B_{2 \mathrm{Bent}}(f)$ that is presented in (8) is added to the right side of (3). Then (3) can be rewritten in form of (9):

$$
\begin{array}{r}
B_{2 \mathrm{Bent}}=\frac{1}{Z_{0 \mathrm{~L}}}\left(\tan \left(\frac{\pi l_{\mathrm{L} 21}}{\lambda_{\mathrm{gL}}(f)}\right)+2 \tan \left(\frac{\pi l_{\mathrm{L} 22}}{\lambda_{\mathrm{gL}}(f)}\right)+\right. \\
\tan \left(\frac{\pi l_{\mathrm{L} 23}}{\lambda_{\mathrm{gL}}(f)}\right)+\tan \left(\frac{\pi l_{\mathrm{L} 31}}{\lambda_{\mathrm{gL}}(f)}\right)+2 \tan \left(\frac{\pi l_{\mathrm{L} 32}}{\lambda_{\mathrm{gL}}(f)}\right)+ \\
\tan \left(\frac{\pi l_{\mathrm{L} 33}}{\lambda_{\mathrm{gL}}(f)}\right) \\
\frac{1}{2 \pi f L_{2}-\left(2 \pi f C_{2}\right)^{-1}}=B_{\mathrm{b} 2}(f)+B_{\mathrm{T}}(f)+B_{2 \mathrm{Bent}}(f) .
\end{array}
$$

The values of $l_{\mathrm{L} 31}=3.2 \mathrm{~mm}, l_{\mathrm{L} 32}=8.1 \mathrm{~mm}$ and $l_{\mathrm{L} 33}=$ $0.9 \mathrm{~mm}$ are considered but the values of $l_{\mathrm{L} 21}=1.3 \mathrm{~mm}$, $l_{\mathrm{L} 22}=1.2 \mathrm{~mm}$ and $l_{\mathrm{L} 23}=1.5 \mathrm{~mm}$ are first estimated. After solving by substituting the cutoff frequency $f_{\mathrm{c}}$ and the attenuation pole frequency $f_{\mathrm{p} 2}$ instead of $f$ in (9) new values for $l_{\mathrm{L} 2}=3.5 \mathrm{~mm}$ and $l_{\mathrm{C} 2}=11.7 \mathrm{~mm}$ are obtained. After determining new values for $l_{\mathrm{L} 2}$ and $l_{\mathrm{C} 2}$, new values $l_{\mathrm{L} 21}=$ $1.1 \mathrm{~mm}, l_{\mathrm{L} 22}=1 \mathrm{~mm}$ and $l_{\mathrm{L} 23}=1.4 \mathrm{~mm}$ can be selected accordingly. Correction the lengths of $l_{\mathrm{L} 4}$ and $l_{\mathrm{C} 4}$ are similar to the method described in this section and could be done by replacing the parameters proportional to the $L_{4} C_{4}$ branch. After the correction process of the $L_{4} C_{4}$ branch, values of $l_{\mathrm{L} 4}=8.5 \mathrm{~mm}$ and $l_{\mathrm{C} 4}=8.3 \mathrm{~mm}$ are obtained.

Figure 4 illustrates EM simulated $S_{21}$ parameters for modified bended layout by (9) for $L_{2} C_{2}$ branch which is shown in Fig. $3 \mathrm{~b}$ (and $L_{4} C_{4}$ branch but not shown), beside $\mathrm{S}_{21}$ parameters for the lumped elements circuit corresponding to connection of $L_{1}, L_{2}, L_{3}$ and $C_{2}$ (or $L_{3}, L_{4}, L_{5}$ and $C_{4}$ for $L_{4} C_{4}$ branch). Also, Figure 4 shows EM simulated $\mathrm{S}_{21}$ parameters for unmodified bended layout of $L_{2} C_{2}$ and $L_{4} C_{4}$

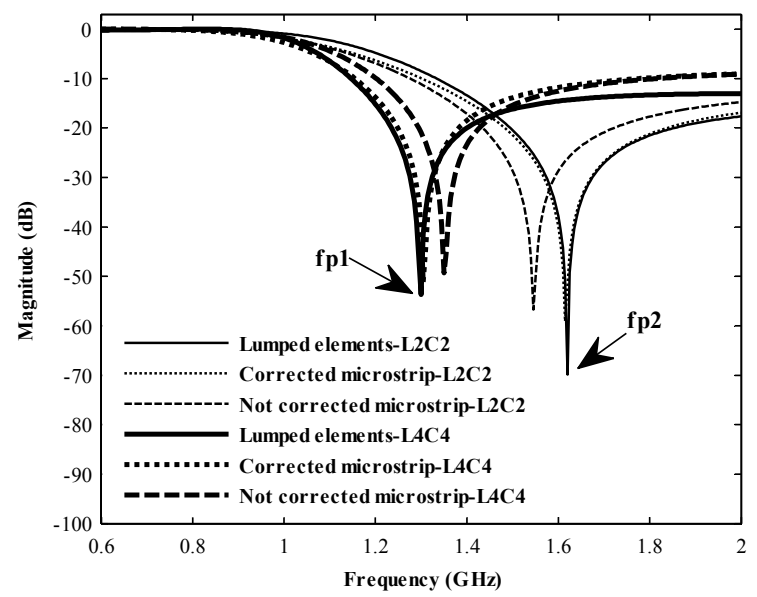

Fig. 4. The $\mathrm{S}_{21}$ parameters of the $L_{2} C_{2}$ or $L_{4} C_{4}$ branches: solid line (or bold solid line) for $L_{2} C_{2}$ (or $L_{4} C_{4}$ ) branch with lumped elements, dot line (or bold dot line) for $L_{2} C_{2}$ (or $L_{4} C_{4}$ ) branch with microstrip lines which bended then corrected with (9), and finally dash line (or bold dash line) for $L_{2} C_{2}$ (or $L_{4} C_{4}$ ) branch with microstrip lines which bended but not corrected with (9). 
branches for comparing. As shown in Fig. 4 the location of $f_{\mathrm{p} 1}$ and $f_{\mathrm{p} 2}$ deviate by bending microstrip high impedance lines from values predicted with (2) however, the correction of the microstrip line lengths by (9) can match the values of $f_{\mathrm{p} 1}$ and $f_{\mathrm{p} 2}$ to values which obtained by (2). The $\mathrm{S}_{21}$ parameters for the corrected bended microstrip layouts and the lumped element circuits are well matched. This match causes the modified LPF cutoff frequency closes to cutoff frequency of the LPF with lumped elements.

\subsection{Adding Suppression Resonators}

It can be seen by looking at Fig. 2 that the microstrip conventional elliptic LPF has a narrow SBW. In order to increase the SBW, a suppression cell consisting of two resonators has been used. Generating transmission zeros (TZs) at frequencies higher than the cutoff frequency can increase the SBW but this action should not interfere with the filter frequency response at frequencies below the cutoff frequency. As shown in Fig. 5, a combination of a radial stub resonator (RSR) and a butterfly stub resonator (BSR) in the middle of $l_{\mathrm{L} 5}$ forms a suppression cell. Now $l_{\mathrm{L} 5}$ is no longer valid and is replaced by $l_{\mathrm{L} 5-1}, l_{\mathrm{L} 5-2}$ and $l_{\mathrm{L} 5-3}$. The lengths of $l_{\mathrm{L} 5-1}, l_{\mathrm{L} 5-2}$ and $l_{\mathrm{L} 5-3}$ are selected by using ADS software tuning application. The values $l_{\mathrm{L} 5-1}=$ $6.5 \mathrm{~mm}, l_{\mathrm{L} 5-2}=6.4 \mathrm{~mm}$ and $l_{\mathrm{L} 5-3}=6.1 \mathrm{~mm}$ is obtained. The RSR structure is shown in Fig. 6. The values of parameters corresponding to Fig. $6 \mathrm{a}$ are $R_{\mathrm{RS}}=7.4 \mathrm{~mm}, r_{\mathrm{RS}}=0.58 \mathrm{~mm}$, $\theta_{\mathrm{RS}}=120^{\circ}$. The equivalent lossless circuit with lumped elements is illustrated in Fig. 6b [22]. Assuming $R_{\mathrm{RS}}<\lambda_{\mathrm{g}} / 8$ and $r_{\mathrm{RS}} \approx R_{\mathrm{RS}} / 10$, the values of $C_{\mathrm{RS}}$ and $L_{\mathrm{RS}}$ are extracted from (10) and (11) [23]:

$$
\begin{gathered}
C_{\mathrm{RS}}=\frac{\theta_{\mathrm{RS}} R_{\mathrm{RS}}^{2} \varepsilon_{\mathrm{eff}}}{240 \pi h c}, \\
L_{\mathrm{RS}}=\frac{120 \pi h\left(2.8-10 r_{\mathrm{RS}} / R_{\mathrm{RS}}\right)}{c \theta_{\mathrm{RS}}}
\end{gathered}
$$

where $\varepsilon_{\text {eff }}$ is effective dielectric constant of microstrip line with width equal to $w_{\mathrm{eq}}=\left(R_{\mathrm{RS}}+r_{\mathrm{RS}}\right) \sin \left(\theta_{\mathrm{RS}} / 2\right)$, also $h=0.813 \mathrm{~mm}$ is dielectric thickness and $c$ is speed of light. As a result, the transmission zero for the RSR (TZ1) can be written as follows:

$$
f_{\mathrm{TZ} 1}=\frac{1}{2 \pi \sqrt{L_{\mathrm{RS}} C_{\mathrm{RS}}}} .
$$

A T-junction is used to connect the RSR to the high impedance microstrip line. Adding the T-junction causes the TZ1 position deviates from the value obtained by (12). To correct adding $\mathrm{T}$-junction effect the $\mathrm{LC}$ equivalent circuit of $\mathrm{T}$-junction is appended to the $\mathrm{LC}$ equivalent circuit of the RSR. The appending of the LC equivalent circuit of the RSR and the LC equivalent circuit of the Tjunction are shown in Fig. 6d.

The values for $\mathrm{T}$-junction are $w_{1 \mathrm{~T}}=0.2 \mathrm{~mm}$ and $w_{2 \mathrm{~T}}=1 \mathrm{~mm}$. The values of $C_{\mathrm{T}}[\mathrm{pF}], L_{1 \mathrm{~T}}$ and $L_{2 \mathrm{~T}}$ are extracted from (13), (14) and (15) with (16) [24], [25]:

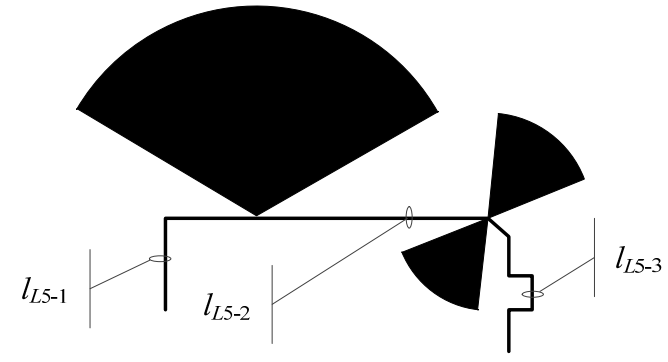

Fig. 5. The RSR and the BSR located at middle of $l_{\mathrm{L} 5}$.

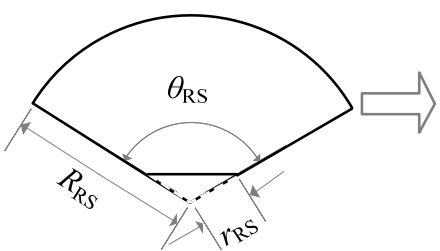

(a)

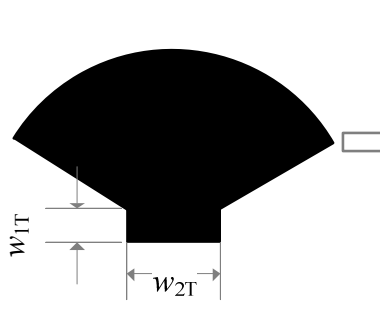

(c)

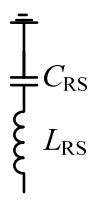

(b)

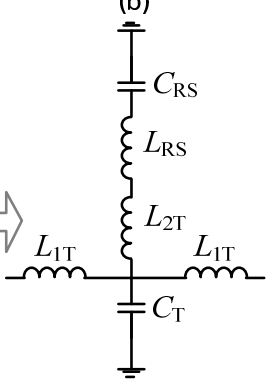

(d)
Fig. 6. The RSR structure and its LC model: (a) The RSR schematic. (b) The LC equivalent circuit of the RSR. (c) The RSR with T-junction schematic. (d) The LC equivalent circuit of the T-junction is appended to the LC equivalent circuit of the RSR.

$$
\begin{gathered}
\frac{C_{\mathrm{T}}}{w_{1 \mathrm{~T}}}=\frac{100}{\tanh \left(0.0072 Z_{0}\right)}+0.64 Z_{0}-261.0 \\
\frac{L_{1 \mathrm{~T}}}{h}=\frac{-w_{2 \mathrm{~T}}}{h}\left[\begin{array}{r}
\frac{w_{2 \mathrm{~T}}}{h}\left(-0.016 \frac{w_{1 \mathrm{~T}}}{h}+0.064\right)+ \\
\left.\frac{0.016 h}{w_{1 \mathrm{~T}}}\right]
\end{array}\right] L_{w, 1}, \\
\frac{L_{2 \mathrm{~T}}}{h}=\left[\begin{array}{c}
\left(\frac{0.12 w_{1 \mathrm{~T}}}{h}-0.47\right) \frac{w_{2 \mathrm{~T}}}{h}+0.195 \frac{w_{1 \mathrm{~T}}}{h}- \\
0.357+0.0283 \sin \left(\frac{\pi w_{1 \mathrm{~T}}}{h}-0.75 \pi\right)
\end{array}\right] L_{w, 2}, \\
L_{w, n}=\frac{Z\left(w_{n \mathrm{~T}}\right) \sqrt{\varepsilon_{\mathrm{eff}}\left(w_{n \mathrm{~T}}\right)}}{c}[\mathrm{nH}]
\end{gathered}
$$

where $Z\left(w_{n \mathrm{~T}}\right)$ and $\varepsilon_{\text {eff }}\left(w_{n \mathrm{~T}}\right)$ are characteristic impedance and effective dielectric constant of $w_{n \mathrm{~T}}\left(w_{1 \mathrm{~T}}\right.$ or $\left.w_{2 \mathrm{~T}}\right)$ respectively.

The values of capacitors and inductors which related to Fig. 6 are brought in Tab. 2. Now the transmission zero for the RSR can be rewritten as follows:

$$
f_{\mathrm{TZ1}}=\frac{1}{2 \pi \sqrt{\left(L_{\mathrm{RS}}+L_{1 \mathrm{~T}}\right) C_{\mathrm{RS}}}} .
$$




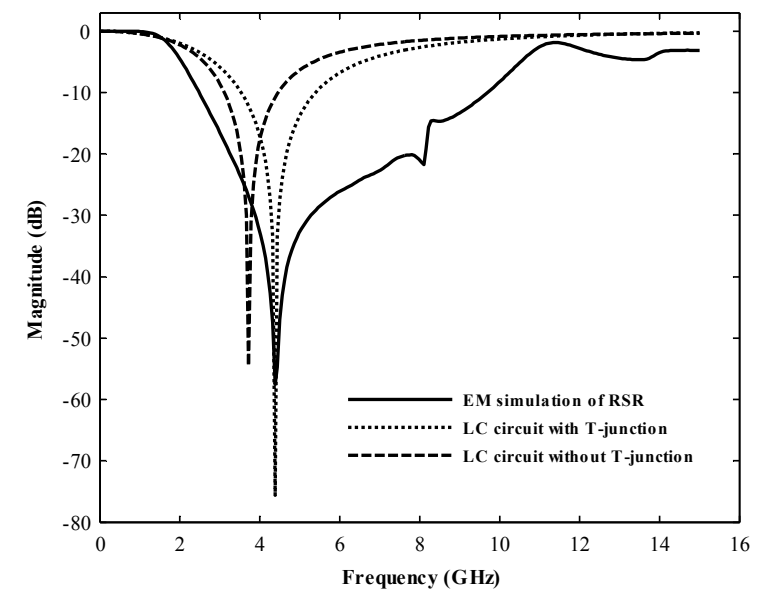

Fig. 7. The solid line shows the $S_{21}$ parameters of the microstrip RSR which is simulated EM; also the dot line and dash line show the $\mathrm{S}_{21}$ parameters of $\mathrm{LC}$ equivalent circuit of the RSR with and without T-junction LC equivalent circuit respectively.

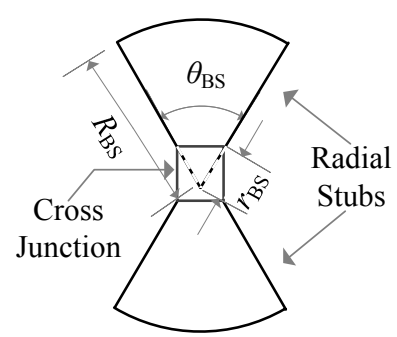

(b)

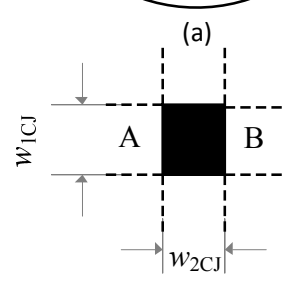

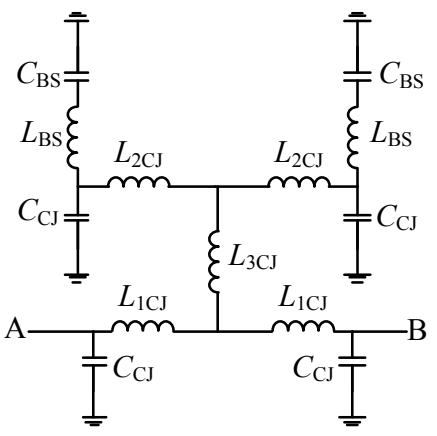

(c)
Fig. 8. The BSR structure and its LC model: (a) The BSR schematic which is created by combining two RSR with one cross junction. (b) The cross junction schematic. (c) The LC equivalent circuit of the BSR.

And $f_{\mathrm{TZ} 1}$ is obtained from (17) with value $4.3 \mathrm{GHz}$. As shown in Fig. 7 the TZ1 in both the $\mathrm{S}_{21}$ parameter curves of the RSR resonator with $\mathrm{T}$-junction and its $\mathrm{LC}$ equivalent circuit is matched. In Fig. 8 the BSR structure is depicted. As shown in Fig. 8a the BSR structure is created by combining two RSR with one cross junction. Also the LC equivalent circuit of the BSR is depicted in Fig. 8c. The values of the $\mathrm{BSR}$ are $R_{\mathrm{BS}}=4 \mathrm{~mm}, r_{\mathrm{BS}}=0.47 \mathrm{~mm}, \theta_{\mathrm{BS}}=$ $65^{\circ}$. The values of cross junction are $w_{1 \mathrm{CJ}}=0.2 \mathrm{~mm}$ and $w_{2 \mathrm{CJ}}=0.51 \mathrm{~mm}$. The value of the parameters of $C_{\mathrm{BS}}$ and $L_{\mathrm{BS}}$ are extracted from (10) and (11) by substituting the RSR parameters with the related BSR parameters. In addition, the calculation of parameters $C_{\mathrm{CJ}}, L_{1 \mathrm{CJ}}, L_{2 \mathrm{CJ}}$ and $L_{3 \mathrm{CJ}}$ is performed using (A1), (A2), (A3) and (A4) [24], [25] which are given in Appendix A. Also, the values of $C_{\mathrm{CJ}}$, $L_{1 \mathrm{CJ}}, L_{2 \mathrm{CJ}}$ and $L_{3 \mathrm{CJ}}$ are brought in Tab. 2 . In next, ignoring the parameter $C_{\mathrm{CJ}}$, the transmission zero of the BSR (TZ2) can be written as:

\begin{tabular}{|c|c|c|c|c|c|c|c|}
\hline Sym. & Value & Sym. & Value & Sym. & Value & Sym. & Value \\
\hline$L_{\mathrm{RS}}$ & 0.9833 & $C_{\mathrm{RS}}$ & 1.858 & $L_{1 \mathrm{~T}}$ & -0.0934 & $L_{2 \mathrm{~T}}$ & -0.2724 \\
\hline$C_{\mathrm{T}}$ & -0.0012 & $L_{\mathrm{BS}}$ & 1.454 & $C_{\mathrm{BS}}$ & 0.2685 & $L_{1 \mathrm{CJ}}$ & 0.0894 \\
\hline$L_{2 \mathrm{CJ}}$ & 0.0490 & $L_{3 \mathrm{CJ}}$ & -0.3135 & $C_{\mathrm{CJ}}$ & -0.0681 & $\varepsilon$ & 3.38 \\
\hline
\end{tabular}

Tab. 2. The values of inductors $[\mathrm{nH}]$ and capacitors $[\mathrm{pF}]$ related to Fig. 6 and Fig. 8.

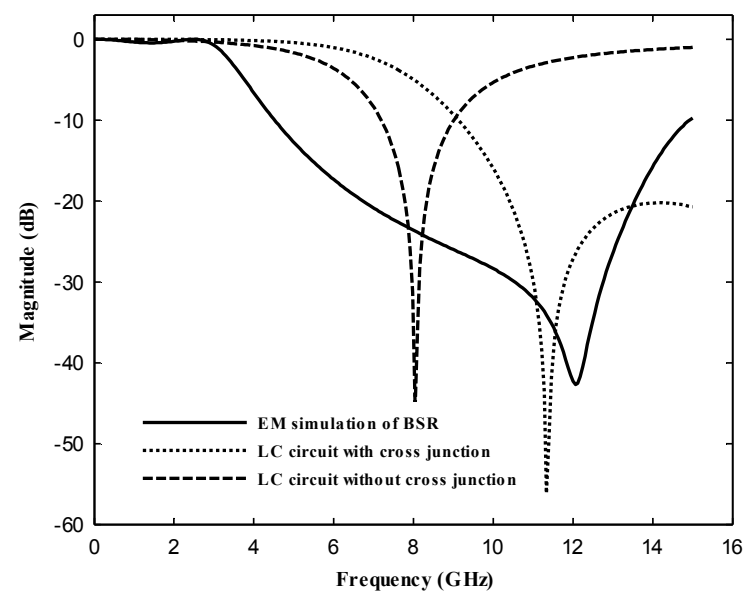

Fig. 9. The solid line shows the $S_{21}$ parameters of the microstrip BSR which is simulated EM; also the dot line and dash line show the $\mathrm{S}_{21}$ parameters of LC equivalent circuit of the RSR with and without cross junction LC equivalent circuit respectively.

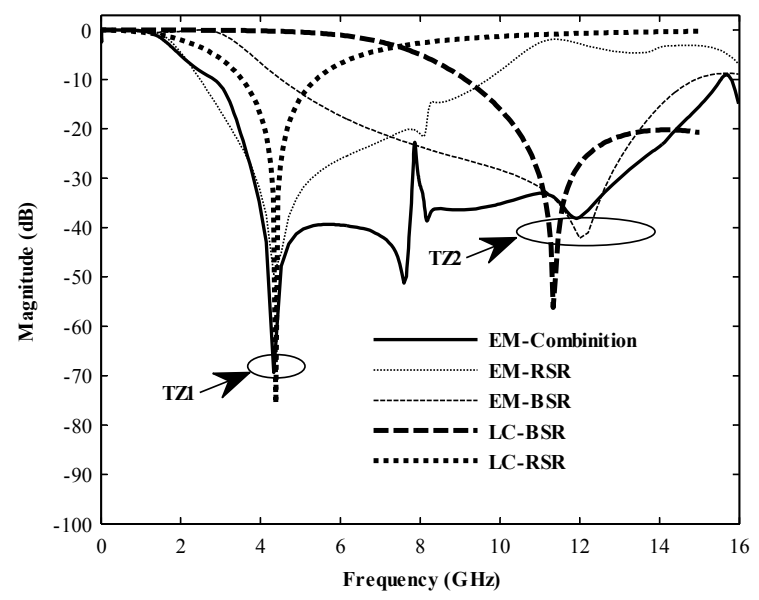

Fig. 10. The $S_{21}$ parameters of the RSR, the BSR and the combination of them which are EM simulated, and the $\mathrm{S}_{21}$ parameters of LC equivalent circuit of the RSR and the BSR.

$$
f_{\mathrm{TZ} 2}=\frac{1}{2 \pi \sqrt{\left(L_{\mathrm{BS}}+L_{2 \mathrm{CJ}}+2 L_{3 \mathrm{CJ}}\right) C_{\mathrm{BS}}}} .
$$

The $f_{\mathrm{TZ} 2}$ is obtained from (18) with value $10.38 \mathrm{GHz}$. This value is near to the $f_{\mathrm{TZ} 2}$ which is obtained from EM simulation of the BSR $(12 \mathrm{GHz})$. As shown in Fig. 9 the TZ2 in both the $S_{21}$ parameter curves of the BSR resonator and its LC equivalent circuit is almost matched.

Also, Figure 10 shows the $\mathrm{S}_{21}$ parameters of the RSR and the BSR resonators separately, combined and those LC equivalent circuits. As shown in Fig. 10 both resonators and combination of them have low IL in the passband. 
These resonators add two transmission zeros at $f_{\mathrm{TZ1}}=$ $4.3 \mathrm{GHz}$ and $f_{\mathrm{TZ2}}=12 \mathrm{GHz}$, but due to their appropriate frequency responses at low frequencies do not appear significant change in the $S_{21}$ parameter in the passband region. Also attenuation of better than $20 \mathrm{~dB}$ of the suppression cell in the frequency range of $3.5 \mathrm{GHz}$ to $14.4 \mathrm{GHz}$ causes the stop bandwidth to be increased appropriately.

\section{Comparing Results of Simulation and Measurement}

Figure 11 shows the final design of the proposed LPF. Most of the values of the parameters related to Fig. 11 were determined in the previous sections however, the newly introduced parameters are $l_{\mathrm{L} 5-11}=3 \mathrm{~mm}, l_{\mathrm{L} 5-12}=$ $3.5 \mathrm{~mm}, l_{\mathrm{L} 5-31}=0.9 \mathrm{~mm}, l_{\mathrm{L} 5-32}=1.5 \mathrm{~mm}, l_{\mathrm{L} 5-33}=1 \mathrm{~mm}, l_{\mathrm{L} 5-34}$ $=0.8 \mathrm{~mm}, \quad l_{\mathrm{L} 5-35}=1.1 \mathrm{~mm}, l_{\mathrm{L} 41}=6.7 \mathrm{~mm}$ and $l_{\mathrm{L} 42}=$ $1.8 \mathrm{~mm}$. The proposed LPF is simulated with the Agilent ADS software. Then the LPF is fabricated on RO4003C substrate with $0.813 \mathrm{~mm}$ thickness, dielectric constant $\left(\varepsilon_{\mathrm{r}}\right)$ equal to 3.38 and loss tangent $(\delta)$ equal to 0.0021 . The fabricated LPF is measured with the Agilent E8362B network analyzer. The actual photograph of the fabricated LPF is shown in Fig. 12. The results of $S_{21}$ and $S_{11}$ parameters simulation and measurement are illustrated in Fig. 13. Based on measurement results, the cutoff frequency is set at $1.12 \mathrm{GHz}$. The $20 \mathrm{~dB}$ attenuation $\mathrm{SBW}$ is obtained 13.4 GHz (from 1.2 to $14.6 \mathrm{GHz}$ ) which it causes the RSB to be equal to 1.7 and the ratio of the SBW to cutoff frequency $\left(\mathrm{SBW} / f_{\mathrm{c}}\right)$ to be equal to 12 . However the IL is less than $0.3 \mathrm{~dB}(0-816 \mathrm{MHz})$ and the $\mathrm{RL}$ is at least $14.4 \mathrm{~dB}$, in addition the $\mathrm{SF}$ is 2.2 .

For six-pole elliptic LPF with lumped elements which is shown in Fig. $1 \mathrm{a}$ the ROF from $3 \mathrm{~dB}$ to $40 \mathrm{~dB}$ is a maximum of about $269 \mathrm{~dB} / \mathrm{GHz}$. For the proposed LPF a good ROF more than $201 \mathrm{~dB} / \mathrm{GHz}$ is obtained which means $75 \%$ of the LPF with lumped elements. In order to achieve a higher ROF, the degree of filter must be larger, which makes the IL unsuitable in addition to filter complexity. Table 3 compares the important parameters of filter with the results of the recent reviewed research. Note that some of compared articles in Tab. 3 have high ROF [4-6] however, compared to the present work in [4] the IL is not appropriate and in [4], [5] the RSB is very small. Also, in [6] the SF is small and other important parameters are not very appropriate. Using the microstrip line bending method the value of the NCS was reduced from $0.255 \lambda_{\mathrm{g}} \times 0.125 \lambda_{\mathrm{g}}$ (for not bended LPF) to $0.147 \lambda_{\mathrm{g}} \times 0.133 \lambda_{\mathrm{g}}$, that means $39 \%$ reduction. As can be deduced from Tab. 3, the method that presented in this paper works well for increasing the SBW and decreasing the NCS, while high ROF is obtained and the other LPF important parameters are in the proper range. It is necessary to mention that ROF $(\zeta)$, SF, RSB and NCS parameters for the LPF are very common and widely used parameters. The ROF is defined as:

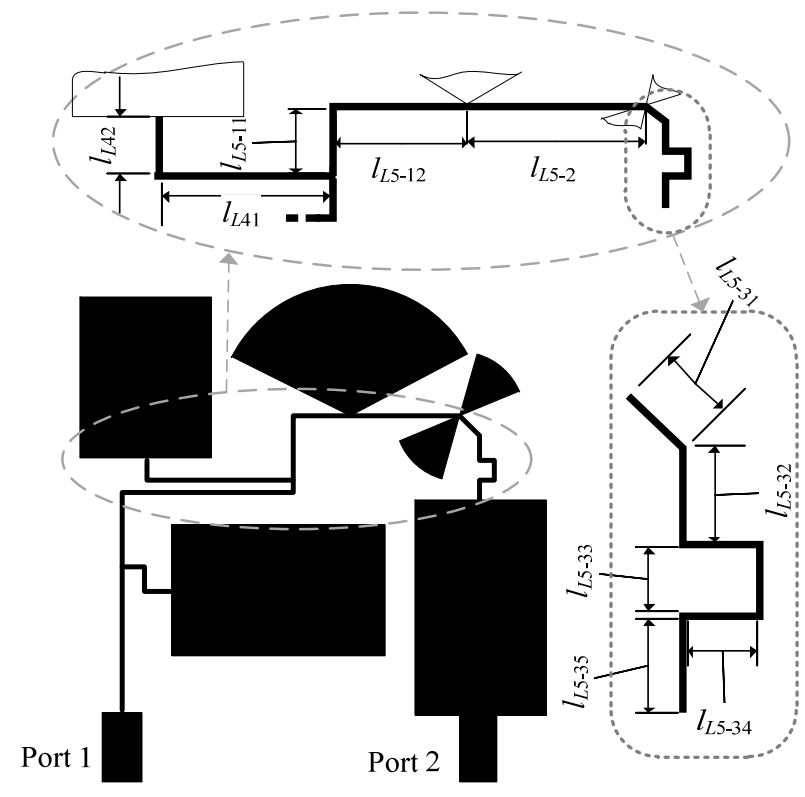

Fig. 11. The layout of final proposed LPF along its remaining details.

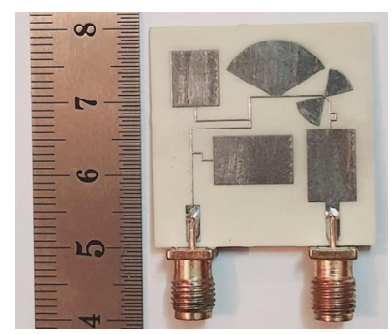

Fig. 12. The actual photograph of the fabricated LPF.

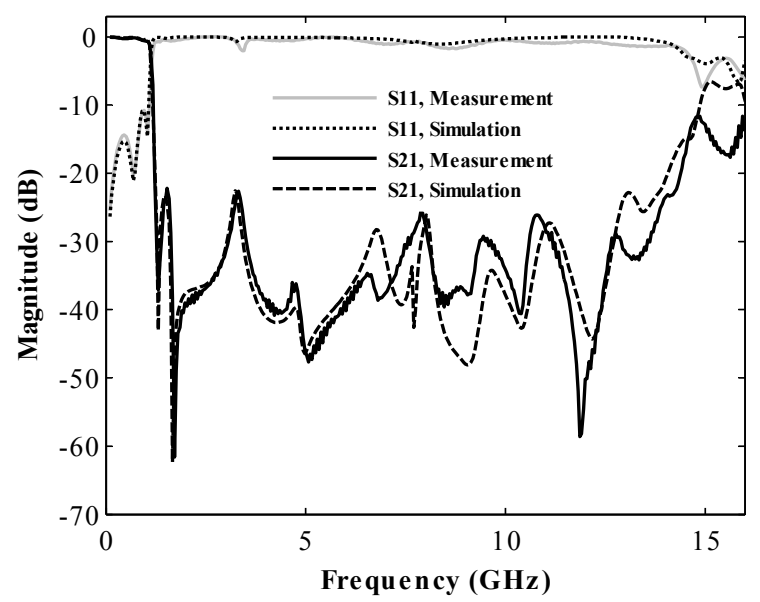

Fig. 13. Comparing the simulated and measured S-parameters for the proposed LPF.

$$
\zeta=\frac{\alpha_{\max }-\alpha_{\min }}{f_{\mathrm{s}}-f_{\mathrm{c}}}
$$

where in this paper $\alpha_{\max }=40 \mathrm{~dB}, \alpha_{\min }=3 \mathrm{~dB}$ and $f_{\mathrm{s}}$ is the first frequency whose attenuation is $40 \mathrm{~dB}$. The SF, the RSB and the NCS are given by (20), (21) and (22) respectively: 


\begin{tabular}{|c|c|c|c|c|c|c|c|}
\hline Ref. & $\boldsymbol{f}_{\mathbf{c}}$ & $\zeta$ & $\mathbf{I L}$ & $\mathbf{R L}$ & SF & RSB & NCS \\
\hline$[3]$ & 1.8 & 228 & - & 17.3 & 2.1 & 1.78 & $0.229 \times 0.140$ \\
\hline$[4]$ & 1.24 & 336 & 0.88 & 14 & 1.93 & 1.33 & $0.100 \times 0.150$ \\
\hline$[5]$ & 1.32 & 411 & - & 17 & 2.8 & 1.00 & $0.158 \times 0.128$ \\
\hline$[6]$ & 1.95 & 440 & 0.4 & 12 & 1.5 & $1.56^{* *}$ & $0.271 \times 0.122$ \\
\hline$[7]$ & 1.64 & 57.8 & - & - & 3.5 & 1.61 & $0.120 \times 0.100$ \\
\hline$[8]$ & 3.8 & 87.3 & 0.1 & 21 & 2.8 & $1.09^{* *}$ & $0.138 \times 0.158$ \\
\hline$[9]$ & 2.68 & - & 0.12 & 18.5 & 2 & $1.51^{* *}$ & $(147.6)$ \\
\hline$[10]$ & 2.8 & 48.5 & 0.1 & 16.5 & 2 & 1.61 & $0.141 \times 0.151$ \\
\hline$[11]$ & 0.9 & $81^{*}$ & 0.5 & 15.5 & $2.24^{* *}$ & $1.78^{* *}$ & $0.176 \times 0.102$ \\
\hline$[12]$ & 2.92 & 35.4 & 0.12 & 16 & 2 & $1.65^{* *}$ & $0.156 \times 0.128$ \\
\hline$[13]$ & 1.44 & 90.2 & 0.2 & 14 & 2 & 1.65 & $(132)$ \\
\hline$[14]$ & 1.52 & 90 & 0.2 & 17 & 2 & 1.66 & $0.062 \times 0.104$ \\
\hline$[15]$ & 1.2 & 135 & 0.35 & 20.6 & 2 & 1.71 & $0.237 \times 0.105$ \\
\hline$[16]$ & 2 & 41 & 0.3 & - & 2 & 1.64 & $0.140 \times 0.100$ \\
\hline$[17]$ & 2.49 & 37.2 & 0.8 & 7.6 & 1.5 & 1.62 & $0.180 \times 0.210$ \\
\hline$[18]$ & 4.2 & 6.14 & 0.5 & - & $1^{* *}$ & 0.749 & $0.417 \times 0.202$ \\
\hline$[19]$ & 1.96 & 104 & 0.6 & 12 & 2.5 & 1.8 & $0.189 \times 0.121$ \\
\hline$[20]$ & 2.2 & $63^{* *}$ & 0.2 & 17.4 & 2 & 1.73 & $0.113 \times 0.107$ \\
\hline$[21]$ & 2.1 & 203 & 0.1 & - & 3.2 & 1.61 & $0.208 \times 0.208$ \\
\hline $\begin{array}{l}\text { This } \\
\text { work }\end{array}$ & 1.12 & 201 & 0.3 & 14.4 & 2.2 & 1.7 & $0.147 \times 0.133$ \\
\hline$*-$ for 60 dB attenuation \\
$* *$ calculated with the information given in the related article \\
\hline
\end{tabular}

Tab. 3. Comparison of $f_{\mathrm{c}}[\mathrm{GHz}], \quad \zeta[\mathrm{dB} / \mathrm{GHz}], \quad \mathrm{IL}[\mathrm{dB}]$, RL $[\mathrm{dB}], \quad \mathrm{SF}, \quad \mathrm{RSB}$ and NCS $\left[\lambda_{\mathrm{g}} \times \lambda_{\mathrm{g}}\right]$ or $\left(\mathrm{mm}^{2}\right)$ parameters between the proposed LPF in this work and the reviewed recent articles.

$$
\mathrm{SF}=\frac{\text { rejection level }[\mathrm{dB}]}{10[\mathrm{~dB}]},
$$

$$
\mathrm{RSB}=\frac{\text { stopband bandwidth }}{\text { stopband center frequency }},
$$

$$
\mathrm{NCS}=\frac{\text { physical size }(\text { length } \times \text { width })}{\lambda_{\mathrm{g}}^{2}} .
$$

\section{Conclusion}

In this paper by using the microstrip lines bending technique the LPF size reduced $39 \%$. In order to match $\mathrm{S}_{21}$ responses between the bended LPF and the lumped elements circuit, the microstrip line lengths were modified mathematically. Adding surplus suppression resonators led to increase the SBW up to 12 times the cutoff frequency. Taking the advantage of the elliptic structure for the LPF caused a high ROF up to $201 \mathrm{~dB} / \mathrm{GHz}$. Low IL, high RL, high SF, simple architecture and low cost are other benefits of the proposed LPF. Finally, close matching results between simulation and measurement represents advantages of using the method proposed in this article.

\section{References}

[1] POZAR, D. M. Microwave Engineering. $2^{\text {nd }}$ ed. New York (USA): John Wiley \& Sons, Inc., 1998. ISBN: 9780471170969

[2] HONG, J.-S., LANCASTER, M. J. Microstrip Filters for RF/Microwave Applications. New York (USA): John Wiley \& Sons, Inc., 2001. DOI: 10.1002/0471221619

[3] ABDIPOUR, AS., ABDIPOUR, AR., KOSRAVI, A. A compact microstrip lowpass filter with ultra-wide rejection band and sharp transition band utilizing combined resonators with triangular patches. Radioengineering, 2018, vol. 27, no. 2, p. 417-424. DOI: $10.13164 /$ re.2018.0417

[4] PHANI KUMAR, K. V., KARTHIKEYAN, S. S. Microstrip lowpass filter with flexible roll-off rates. $A E U$ International Journal of Electronics and Communications, 2018, vol. 86, p. 63-68. DOI: 10.1016/j.aeue.2018.01.025

[5] ROSHANI, S., GOLESTANIFAR, A., GHADERI, A., et al. High performance microstrip low pass filter for wireless communications. Wireless Personal Communications, 2017, vol. 99, no. 1, p. 497-507. DOI: 10.1007/s11277-017-5123-1

[6] KUMAR, L., PARIHAR, M. S. A wide stopband low-pass filter with high roll-off using stepped impedance resonators. IEEE Microwave and Wireless Components Letters, 2018, vol. 28, no. 5 , p. 404-406. DOI: $10.1109 / 1 m w c .2018 .2816520$

[7] ZHANG, B., LI, S., HUANG, J. Compact lowpass filter with wide stopband using coupled rhombic stubs. Electronics Letters, 2015, vol. 51, no. 3, p. 264-266. DOI: 10.1049/e1.2014.3490

[8] RAMANUAJAM, P., RAMESH VENKATESAN, P. G., ARUMUGAM, C. Miniaturized low-pass filter design with wide stopband using complementary split-ring resonator. Microwave and Optical Technology Letters, 2019, vol. 61, no. 12, p. 2832-2837. DOI: 10.1002/mop.31951

[9] SHEIKHI, A., ALIPOUR, A., ABDIPOUR, A. Design of compact wide stopband microstrip low-pass filter using T-shaped resonator. IEEE Microwave and Wireless Components Letters, 2017, vol. 27, no. 2, p. 111-113. DOI: 10.1109/1mwc.2017.2652862

[10] SHEIKHI, A., ALIPOUR, A., HEMESI, H. Design of microstrip wide stopband lowpass filter with lumped equivalent circuit. Electronics Letters, 2017, vol. 53, no. 21, p. 1416-1418. DOI: $10.1049 / \mathrm{el} .2017 .1715$

[11] CHEN, F. C., LI, R. S, CHU, Q. X. Ultra-wide stopband low-pass filter using multiple transmission zeros. IEEE Access, 2017, vol. 5, p. 6437-6443. DOI: 10.1109/access.2017.2693344

[12] HAYATI, M., SHAMA, F. A compact lowpass filter with ultra wide stopband using stepped impedance resonator. Radioengineering, 2017, vol. 26, no. 1, p. 269-274. DOI: $10.13164 /$ re.2017.0269

[13] EKHTERAEI, M., HAYATI, M., KAZEMI, A. H., et al. Design and analysis of a modified rectangular-shaped lowpass filter based on LC equivalent circuit. AEU - International Journal of Electronics and Communications, 2020, vol. 126, p. 1-9. DOI: $10.1016 /$ j.aeue. 2020.153290

[14] LOTFI, S., HAYATI, M. Compact low-pass filter with ultra-wide stopband using analysed triangular-shaped resonator. Electronics Letters, 2017, vol. 53, no. 15, p. 1050-1052. DOI: $10.1049 / \mathrm{el} .2017 .1169$

[15] SEN, S., MOYRA, T. Compact low-cost microstrip lowpass filter with sharp roll-off and wide attenuation band. International 
Journal of RF and Microwave Computer-Aided Engineering, 2019, vol. 29, no. 11. DOI: $10.1002 /$ mmce. 21917

[16] SEN, S., MOYRA, T. A compact lowpass filter using interdigital line resonator with wide stopband. Iranian Journal of Science and Technology, Transactions of Electrical Engineering, 2019, vol. 43, no. 3, p. 469-478. DOI: 10.1007/s40998-019-00191-w

[17] SHI, L., FAN, Z., XIN, D. Miniaturized low-pass filter based on defected ground structure and compensated microstrip line. Microwave and Optical Technology Letters, 2019, vol. 62, no. 3, p. 1093-1097. DOI: 10.1002/mop.32144

[18] SINGHAL, D., SINGH, S., KAUSHAL, V., et al. Wide band stop response using interdigital capacitor/CSRR DGS in elliptical microstrip low-pass filter. Journal of Microwaves, Optoelectronics and Electromagnetic Applications, 2020, vol. 19, no. 4, p. 495-509. DOI: 10.1590/2179-10742020v19i4945

[19] JIANG, S., XU, J. Sharp roll-off planar lowpass filter with ultra-wide stopband up to $40 \mathrm{GHz}$. Electronics Letters, 2017, vol. 53, no. 11, p. 734-735. DOI: 10.1049/el.2017.1238

[20] SHAMA, F., HAYATI, M., EKHTERAEI, M., et al. Compact microstrip lowpass filter using meandered unequal T-shaped resonator with ultra-wide rejection band. AEU - International Journal of Electronics and Communications, 2018, vol. 85, p. $78-83$. DOI: $10.1016 /$ j.aeue.2017.12.038

[21] ABDIPOUR, AS., ABDIPOUR, AR., ALAHVERDI, M. A design of microstrip lowpass filter with wide rejection band and sharp transition band utilizing semi-circle resonators. Radioengineering, 2018, vol. 27, no. 4, p. 1043-1049. DOI: 10.13164/re.2018.1043

[22] GIANNINI, F., PAOLONI, C., RUGGII, M. CAD-oriented lossy models for radial stubs. IEEE Transactions on Microwave Theory and Techniques, 1988, vol. 36, no. 2, p. 305-313. DOI: $10.1109 / 22.3519$

[23] KWON, H., LIM, H., KANG, B. Design of 6-18 GHz wideband phase shifters using radial stubs. IEEE Microwave and Wireless Components Letters, 2007, vol. 17, no. 3, p. 205-207. DOI: $10.1109 / 1 \mathrm{mwc} .2006 .890481$

[24] WADELL, B. C. Transmission Line Design Handbook. Norwood (USA): Artech House, 1991. ISBN: 0890064369

[25] GARG, R., BAHL, I. J. Microstrip discontinuities. International Journal of Electronics, 1978, vol. 45, no. 1, p. 81-87. DOI: $10.1080 / 00207217808900883$

\section{About the Authors ...}

Abbas HOSEINABADI received his B.Sc. and M.Sc. from Electronics Department of Arak branch, Islamic Azad University. He is currently a Ph.D. student at Electronics Department of Saveh branch, Islamic Azad University.

Mohammad Bagher TAVAKOLI received his $\mathrm{Ph} . \mathrm{D}$. in Electronics from Science and Research branch, Islamic Azad University in 2013 and his M.Sc. and B.Sc. from Electronics Department of Arak branch, Islamic Azad University. He is currently an Associate Professor at the Islamic Azad University of Arak and the Dean of the Faculty of Engineering in Islamic Azad University of Arak.

Mohammad Jalal RASTEGAR FATEMI received the M.Sc. degree from Azad University of Arak 2006 and the Ph.D. degree in the Dept. of Electrical Engineering, University of Science and Research of Tehran 2011, both in Electronic Engineering. He joined the Azad University of Saveh in 2006 as lecturer. His research interests focus on power electronics and semiconductor device modeling and he does some researches on image processing and computer vision.

Farbod SETOUDEH received his Ph.D. degree in Electrical Engineering from Science and Research Branch of Azad University and is currently an Assistant Professor at Electrical Engineering Department of Arak University of Technology. His research interests include intelligent system, signal processing, and nonlinear system.

\section{Appendix A: Computational Equations of $C_{\mathrm{CJ}}, L_{1 \mathrm{CJ}}, L_{2 \mathrm{CJ}}$ and $L_{3 \mathrm{CJ}}$}

In this appendix, the computational equations for the LC equivalent circuit of cross junction (A1), (A2), (A3) and (A4) are presented. All necessary parameters and the results of calculation have been given in Sec. 2.3.

$$
\begin{gathered}
\frac{C_{\mathrm{CJ}}}{w_{1 \mathrm{CJ}}}=\left\{\left[\frac{86.6 w_{2 \mathrm{CJ}}}{h}-30.9 \sqrt{\frac{w_{2 \mathrm{CJ}}}{h}}+367.0\right] \log \left(\frac{w_{1 \mathrm{CJ}}}{h}\right)+\left(\frac{w_{2 \mathrm{CJ}}}{h}\right)^{3}+\frac{74.0 w_{2 \mathrm{CJ}}}{h}+130.0\right\}\left(\frac{w_{1 \mathrm{CJ}}}{h}\right)^{-1 / 3}- \\
\frac{L_{1 \mathrm{CJ}}}{h}=\left\{\left[\frac{165.6 w_{2 \mathrm{CJ}}}{h}+31.2 \sqrt{\frac{w_{2 \mathrm{CJ}}}{h}}-11.8\left(\frac{w_{2 \mathrm{CJ}}}{h}\right)^{2}\right] \frac{w_{1 \mathrm{CJ}}}{h}-\frac{32.0 w_{2 \mathrm{CJ}}}{h}+3.0\right\}\left(\frac{w_{1 \mathrm{CJ}}}{h}\right)^{-3 / 2}, \\
\frac{L_{2 \mathrm{CJ}}}{h}=\left\{\left[\frac{165.6 w_{1 \mathrm{CJ}}}{h}+31.2 \sqrt{\frac{w_{1 \mathrm{CJ}}}{h}}-11.8\left(\frac{w_{1 \mathrm{CJ}}}{h}\right)^{2}\right] \frac{w_{2 \mathrm{CJ}}}{h}-\frac{32.0 w_{1 \mathrm{CJ}}}{h}+3.0\right\}\left(\frac{w_{2 \mathrm{CJ}}}{h}\right)^{-3 / 2}, \\
\frac{L_{3 \mathrm{CJ}}}{h}=337.5+\left(1+\frac{7 h}{w_{1 \mathrm{CJ}}}\right) \frac{h}{h}-\frac{5.0 w_{2 \mathrm{CJ}}}{h} \cos \left[\frac{\pi\left(1.5 h-w_{1 \mathrm{CJ}}\right)}{2 h}\right] \cdot
\end{gathered}
$$

